\title{
EVALUATION OF FRICTIONAL RESISTANCE FOR DIFFERENT TYPES OF TOOTH COLORED ORTHODONTIC BRACKETS (AN IN-VITRO STUDY)
}

\author{
Ahmed Mohamed Hussein Hassanen ${ }^{1 *}$, Saad Eldin Sadek Elgezawy ${ }^{2}$, Mohammed Abbas Masoud $^{3}$
}

\begin{abstract}
Objective: Friction in orthodontic treatment believed to reduce the efficiency of orthodontic appliances during sliding mechanics. During sliding mechanics, a friction force is produced in the bracket arch-ligature unit tends to counteract the applied force and, in turn, resists the desired movement. Thus the objective was to compare the frictional properties of three different types of tooth colored brackets with arch wire. Material and methods: A total of 21 conventionally ligated first premolar aesthetic brackets were used in this study. The samples were divided into 3 main groups (7 each) according to bracket material: group (I): Plastic Bracket with metal slot; group (II): monocrystalline alumina bracket; group (III): polycrystalline alumina bracket. Single upper first premolar brackets were mounted on a custom-made acrylic fixture and straight section of rectangular stainless steel arch wire $0.019^{\prime \prime} \times 0.025^{\prime \prime}$ were ligated to the bracket with conventional elastomeric ligature. Frictional resistance was measured with an Instron universal testing machine. The data was statistically analyzed using one-way ANOVA and Tukey's pairwise tests. Results: Statistical results showed that FR generated by the plastic bracket (307.7 \pm 23.9 gr)with metal slot was significantly lower than that generated with the ceramic bracket (mono and polycrystalline ceramic) and showed the lowest FR with the SS wires ,this was followed by polycrystalline ceramic bracket(366.6 $53.9 \mathrm{gr})$ which generated smaller amount of FR but relatively on the higher side when compared with plastic bracket with metal slot .Monocrystalline bracket generated the maximum amount of FR

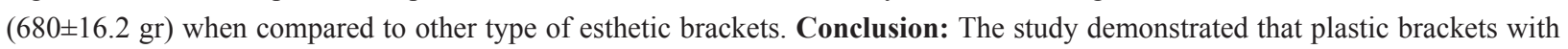
metal slot showed the least frictional resistance of the three-type bracket used.
\end{abstract}

KEYWORDS: Frictional resistance, monocrystalline ceramic brackets, polycrystalline ceramic bracket and plastic brackets with metal slot.

\section{INTRODUCTION}

With respect to orthodontic care, the appearance of fixed orthodontic devices has always been of particular concern, as the number of adults seeking orthodontic treatment has increased. In the 1970s, the use of plastic brackets that were injection molded from the aromatic polymer polycarbonate included attempts to manufacture brackets from various aesthetic materials. Documented problems included crazing a and deformation (1). These problems were not also altered by alternative composite brackets made of chopped glass fibers. For orthodontic uses, it was approximately 20 years until ceramic brackets were available. The ceramic brackets available today are either in polycrystalline

1. 1 Master's candidate, Department of Dental Bio-Material Faculty of Dental Medicine, Al-Azhar University, Cairo (Boys)

2. Assistant Professor, Bio-Materials Department, Faculty of Dental Medicine, Al-Azhar University, Cairo (Boys).

3. Assistant Professor, Bio-Materials Department, Faculty of Dental Medicine, Al-Azhar University, Cairo (Boys).

-Corresponding author: denthassanen@gmail.com 
or monocrystalline shapes, made of alumina $\left(\mathrm{Al}_{2} \mathrm{O}_{3}\right)$. A purer structure, a smooth structure, is the development phase of monocrystalline brackets., and a considerably harder substance than the fabrication of polycrystalline brackets ${ }^{(1)}$. The majority of studies performed on ceramic brackets not only reported the problems of coloring and early torque fracture ${ }^{(2)}$, but also showed increased ceramic bracket friction compared to metal brackets. ${ }^{(3)}$.

Friction at the bracket-wire interface can prevent the achievement of optimal force levels in the supporting tissues during mechanotherapy involving movement of the bracket relative to the wire. The hard and soft tissue response therefore appears to benefit from a decrease in frictional resistance. It has been suggested that about 50 percent of the force applied to a tooth slide is used to counteract friction ${ }^{(4)}$. Other factors influencing frictional resistance include saliva, the dimension and material of the arch wire ${ }^{(5)}$, wire angulation to the bracket and ligation mode ${ }^{(4,6)}$.

Many researchers (4-8) have shown increased frictional resistance, especially with polycrystalline ceramic ${ }^{(9)}$ and plastic brackets. Therefore, aesthetic brackets with metal slots were developed to decrease the frictional forces and overcome these drawbacks of ceramic materials ${ }^{(10)}$.

Thus, the purpose of this study will be directed to evaluate the frictional resistance changes of tooth-colored brackets. The null hypothesis that will be tested, no difference between different esthetic brackets regarding to friction resistance.

\section{MARTIAL AND METHODS}

A total of 21 conventionally ligated first premolar aesthetic brackets (.022-in slot size Roth prescription) were used in this study. The samples were divided into 3 main groups (7 each) according to bracket material: group (I): Plastic Bracket with metal slot (ok real resin, Hubit, Korea), group(II): monocrystalline alumina bracket (Perfect Clear II, Hubit, Korea), group (III): polycrystalline alumina bracket (Unitek Gemini Clear Brackets, 3M, California). Three aesthetic brackets are listed in table [1] and shown in figures [1]. Single upper first premolar brackets were mounted on a custom-made acrylic fixture and straight section of rectangular stainless steel (SS) archwire, .019x .025 , were ligated to the bracket with conventional elastomeric ligature.

TABLE (1) Grouping of samples for the study.

Group I: Plastic Bracket with metal slot (ok real resin, Hubit, Uiwang, Korea $\quad(n=7)$.

Group II: monocrystalline alumina bracket (Perfect Clear II, Hubit, Uiwang, Korea) $\quad(n=7)$.

Group III:polycrystalline alumina bracket

(Unitek Gemini Clear Brackets,3M,California)(n=7)

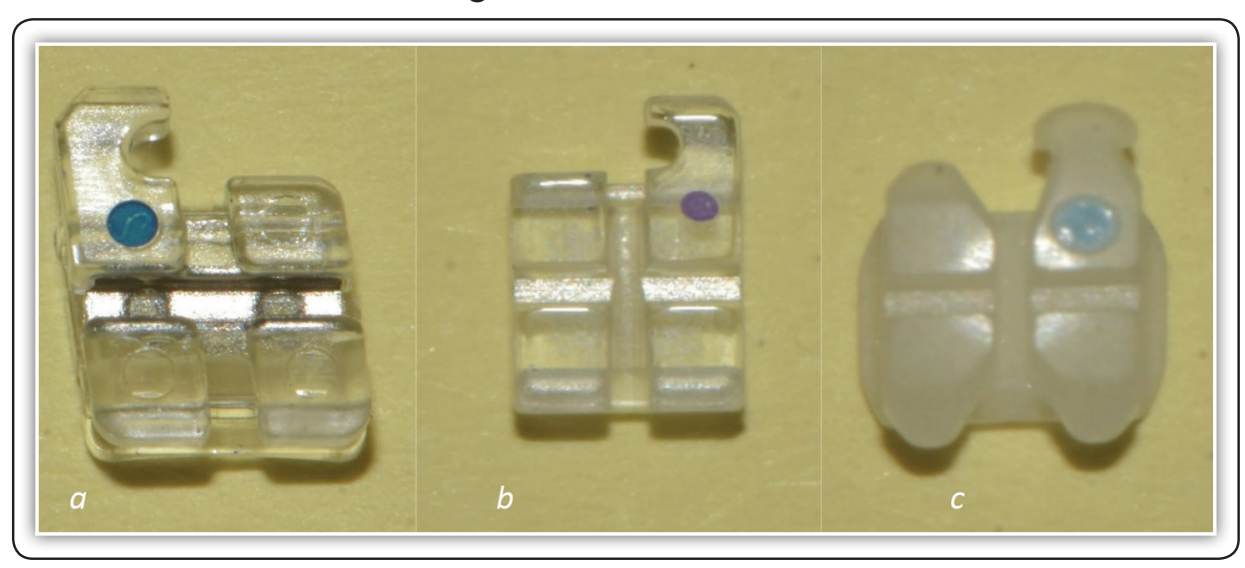

FIG (1) The tested bracket groups: a Plastic Bracket with metal slot, b monocrystalline alumina ceramic brackets, and c: polycrystalline alumina bracket. 
Mold construction: A specially designed metal mold was fabricated to form rectangular specimens with dimensions of $(40 \mathrm{~mm}$ length, $25 \mathrm{~mm}$ width, and $5 \mathrm{~mm}$ height). These dimensions were determined according to the recommendation of international standards organization ISO NO.4049 (2000).

To evaluate the frictional_resistance of the aesthetic brackets 21 test samples were prepared. The monocrystalline ceramic brackets polycrystalline ceramic bracket and Plastic Bracket with metal slotwere used in this study. They were divided into three main groups 10 for each; according to the type of bracket material $(n=7)$. Group I used Plastic Bracket with metal slot Group II used the monocrystalline ceramic brackets and Group III used the polycrystalline ceramic bracket and Group. frictional resistance between bracket and wire was measured.

Teeth selection: Thirty freshly extracted human premolar teeth were used in this study. Teeth were examined to make sure that they are free of caries, cracks and hypoplastic defect. All teeth were handscaled to remove calculus and soft tissues. Selected teeth were stored at room temperature in distilled water.

Preparation of the acrylic block: The split mold was positioned over a glass slab and a polyester strip to avoid adhesion with the unpolymerized material then filled with self-curing acrylic resin (acrostone dental factor, England), then the teeth, including the roots were horizontal completely embedded till the cervical constriction of crown.

Stainless steel spatula used to removal of excess before polymerization, taking care to minimize the entrapped air after complete polymerization of the acrylic resin, the tooth in the set acrylic resin was removed from the mould and finished and stored in distilled water at room temperature.

Bonding of brackets procedures: The samples were divided into three main groups $(n=7)$.
According to manufacturer instructions for each bracket type, the enamel surface of each specimen was etched with 37\% phosphoric acid (fine etch 37 gel type, Spident Inc., Republic of Korea)) for 30 seconds, rinsed with water for 20 seconds, and dried with absorbent paper. Then the adhesive (Adoper single Bond 2 Light cured bond 3M ESPE St.Paul,MN USA)was applied using a saturated brush tip and lightly air-dried for 2 seconds, and light-cured for 10 seconds with LED curing unit 3M ESPE Elipar Deep Cure L Light Cure (3M ESPE, St Paul, MN, USA) with a light output of $1000 \mathrm{mw} \backslash$ $\mathrm{cm} 2$ according to the manufacturer instructions

Then the light cure paste composite (Filtek Z350 Xt 3M ESPE St.Paul, MN, USA) appplied using plastic applicator into the bracket base by firmly smearing the paste into the mesh. Placed on the tooth surface and press firmly to achieve tight contact between bracket and tooth surface then removed any excess composite around bracket periphery by explorer probe. Each side of bracket was light-cured for 20 seconds with LED curing unit with a light output of $1000 \mathrm{~mW} \backslash \mathrm{cm} 2$. according to the manufacturer instructions. The samples were then stored in distilled water at $37 \mathrm{C}$ for $24 \mathrm{~h}$.

Stainless steel (SS) Orthodontic wire used was manufactured by (Denturam Co. Germany), with a cross-section $0.019^{\prime \prime} \times 0.025^{\prime \prime}$. were ligated into the bracket slots using the Conventional Elastomeric Ligatures (American Orthodontics, Sheboygan, WI, USA).

The universal testing machine (Instron Corporation, Canten Industries, Inc., St. Petersburg, Florida, USA) was used for measuring the generated frictional force at the bracket-wire interface by sliding the wire through the bracket slot under small tangential displacements. The acrylic block was fixed firmly on the universal testing machine (Figure 2). Then, posterior sections of each wire $4 \mathrm{~cm}$ long were inserted into the bracket slot. 


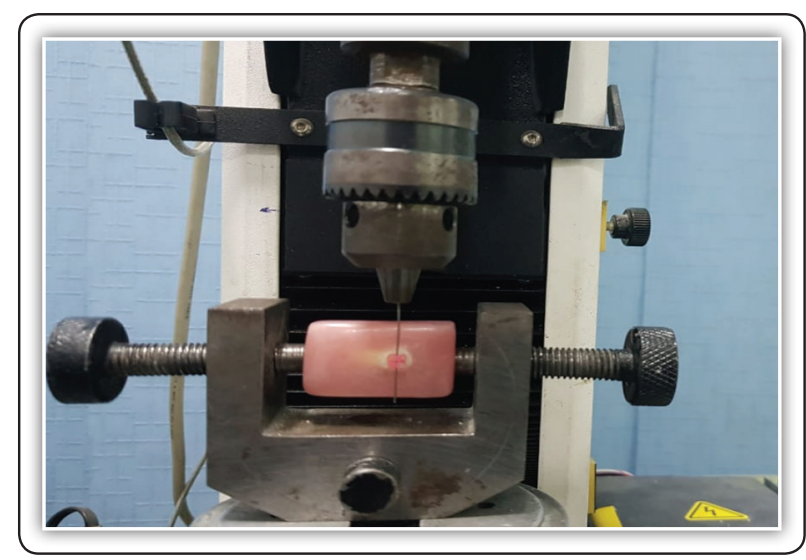

FIG (2) The acrylic block was fixed on the universal testing machine ( Instron Corporation, Canten Industries, Inc., St. Petersburg, Florida, USA).

Prior to testing to remove any residue or debris, all tested wire segments and brackets were washed with isopropyl alcohol. Elastomeric ligatures(American Orthodontics, Sheboygan, WI, USA) were put over the bracket tie wings engaging the arch wire .One end of the test wire was left free, and the other end was tightly connected to the moving upper arm of the $5 \mathrm{~kg}$ load cell universal testing machine.

At a speed of $5 \mathrm{~mm} / \mathrm{min}$ for $1 \mathrm{~min}$, the tested wire was pulled upward through the bracket slot. During clamping, care was taken to avoid introducing torsion into the checked wire. After the wire was lightly tightened and was completely in a straight, vertical position towards the moving arm, the reading was set to give a zero reading (upper fixture). This meant that only the frictional force was registered as the only force transmitted by the moving arm to the wire and through the checked. In grams, the frictional force produced by each wire-bracket assembly was recorded. The block was removed after each test and a new specimen with a new ceramic bracket was put in place. In the bracket's slot, a new wire specimen was fitted. Then the mold was firmly placed on the testing machine, and for various arch wire specimens, the procedure was repeated. Before each measuring session, the load cell was adjusted. While the wire was drawn the same distance through different bracket-arch wire assemblies, it registered the static frictional force. The static frictional force was determined as the force value necessary to begin the movement of the wire through the bracket slot. This force was measured as the maximal initial moving force on the universal testing machine digital display at 1 min intervals of moving wire.

\section{RESULTS}

Static and kinetic friction force (Gram) results (mean \pm SD) for each bracket group were presented in table (2) and graphically drawn in figure (3).

\section{Static friction force:}

The highest mean \pm SD values of static friction force were recorded for Gr_II $(692.3 \pm 25.4$ gr $)$ followed by Gr_III mean \pm SD values $(413.7 \pm 27.1$ gr), meanwhile the lowest mean $\pm \mathrm{SD}$ value was recorded with Gr_I (368.2 \pm 40.5 gr). The difference between groups was statistically significant as indicated by one way ANOVA followed by Tukey's pair-wise post-hoc test $(\mathrm{F}=214.1, \mathrm{P}=<0.0001<0.05)$ as shown in in table (1) and figure (2).

\section{Kinetic friction force;}

The highest mean $\pm \mathrm{SD}$ values of static friction force were recorded for Gr_II ; $(680 \pm 16.2$ gr $)$ followed by Gr_III mean \pm SD values $(366.6 \pm 53.9$ gr) meanwhile the lowest mean $\pm \mathrm{SD}$ value was recorded with Gr_I (307.7 \pm 23.9 gr). The difference between groups was statistically significant as indicated by one way ANOVA followed by Tukey's pair-wise post-hoc test $(\mathrm{F}=224.5, \mathrm{P}=<0.0001<0.05)$ as shown in in table (2) and figure (3). 
TABLE (2) Static and kinetic friction force (Gram) results (mean \pm SD) for each bracket group

\begin{tabular}{|c|c|c|c|c|c|c|c|c|c|}
\hline \multirow{4}{*}{\multicolumn{2}{|c|}{ Variables }} & \multicolumn{8}{|c|}{ Friction force } \\
\hline & & \multicolumn{4}{|c|}{ Static } & \multicolumn{4}{|c|}{ Kinetic } \\
\hline & & \multirow{2}{*}{ Mean } & \multirow{2}{*}{$\mathrm{SD}$} & \multicolumn{2}{|c|}{$95 \% \mathrm{CI}$} & \multirow{2}{*}{ Mean } & \multirow{2}{*}{ SD } & \multicolumn{2}{|c|}{$95 \% \mathrm{CI}$} \\
\hline & & & & Lower & Upper & & & Lower & Upper \\
\hline \multirow{3}{*}{$\begin{array}{l}\text { Bracket } \\
\text { groups }\end{array}$} & Gr_I & $368.2 \mathrm{C}$ & 40.5 & 330.7 & 405.7 & $307.7 \mathrm{C}$ & 23.9 & 285.6 & 329.9 \\
\hline & Gr_II & $692.3 \mathrm{~A}$ & 25.4 & 668.8 & 715.9 & $680 \mathrm{~A}$ & 16.2 & 664.9 & 695 \\
\hline & Gr_III & 413.7B & 27.1 & 388.7 & 438.8 & $366.6 \mathrm{~B}$ & 53.9 & 316.7 & 416.5 \\
\hline \multirow{2}{*}{ Statistics } & \multirow{2}{*}{ ANOVA } & \multicolumn{2}{|c|}{ F value } & \multicolumn{2}{|c|}{214.1} & \multicolumn{2}{|c|}{ F value } & \multicolumn{2}{|c|}{224.5} \\
\hline & & \multicolumn{2}{|c|}{ P value } & \multicolumn{2}{|c|}{$<0.0001 *$} & \multicolumn{2}{|c|}{ P value } & \multicolumn{2}{|c|}{$<0.0001 *$} \\
\hline
\end{tabular}

Different superscript capital letter in the same column indicating statistically significant difference between groups ( $p<0.05$ ) CI; confidence intervals $\quad$ *; significant $(p<0.05)$ ns; non-significant $(p>0.05)$

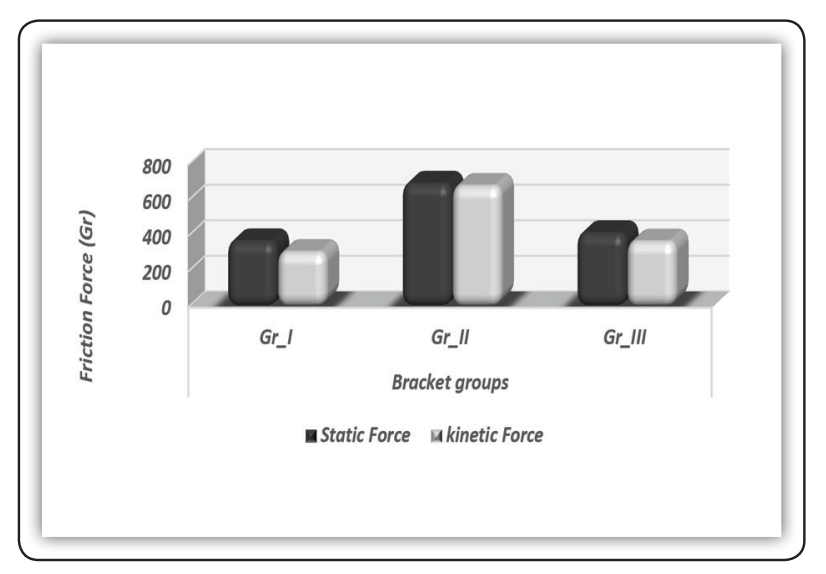

FIG (2) Column chart of the mean values of static and kinetic friction force results for each bracket group

\section{DISCUSSION}

Friction refers to a force that occurs every time two objects are rubbed against each other. The interactive force which have elements that are both perpendicular and tangent to the surface when the surfaces of two bodies are in contact. The force applied is the perpendicular component, and the friction force is the tangential component. The frictional force works in two opposite directions of this movement if there is relative slipping at the floor. This ratio of tangential force to force applied during sliding is known as the friction coefficient and depends on the existence of the two contact surfaces ${ }^{(11)}$.

To a large degree, the effectiveness of orthodontic tooth movement with pre-adjusted appliances depends on the orthodontic archwire's ability to slide through brackets and tubes. With the use of sliding mechanics, the major drawback is the friction caused during orthodontic tooth movement between the bracket and the arch wire. Orthodontists must select a frictionless device for efficiency in sliding mechanics ${ }^{(12)}$.

Kusy and Whitley partitioned the resistance to tooth movement into three separate components. ${ }^{(3)}$. The first component is classical friction that occurs between the wire and bracket surfaces and is further divided into static and kinetic friction. The second component is binding that occurs when a tooth is tipped or a wire is flexed so that the wire contacts the corner of the bracket. The third component is notching ${ }^{(13)}$.

For purpose of standardization in the present study the brackets in this study were chosen to be with zero degrees of tip and torque to allow the 
only friction present to be classical friction and not due to binding or notching. Similarly, the wire was kept constant in terms of material and size to allow the bracket material to be the only variable among the three components. The arch wire used was $0.019 " \times 0.025$ " stainless steel $[\mathrm{SS}]$ wire. This wire dimension was chosen because It is known that the frictional force tends to increase with rectangular cross-section wires in comparison with round wires. Cacciafesta et al ${ }^{(14)}$ reported higher frictional force with an increase in orthodontic arch wire thickness.

The results of the current study show that there was statistically significant difference between different types of brackets regardless the type of material .monocrystalline Ceramic brackets showed the statistically significant highest mean frictional forces, followed by polycrystalline ceramic bracket $(366.6 \pm 53.9$ gr) which generated smaller amount of FR but Relatively on the higher side when compared with plastic bracket with metal slot .monocrystalline bracket Generated the maximum amount of FR; $(680 \pm 16.2$ gr) when compared to other type of esthetic brackets.

The results of the present study that concur with several studies reporting higher frictional forces in brackets versus polycrystalline ceramic brackets or brackets with metal slot $(8,15,16,17)$. Nevertheless, those studies were inconsistent when comparing the two types of ceramic brackets. Some investigators stated that monocrystalline ceramic brackets generate lower frictional resistance than polycrystalline ceramic brackets ${ }^{(18)}$, while others reported no differences between the two types ${ }^{(19)}$.

Many investigators have attributed high friction in ceramic brackets to their rough surface $(13,20,21,22)$. Contrary to what one might expect, higher frictional resistance does not correlate with a rougher slot surface. In fact, the relationship between the bracket's surface roughness and frictional characteristics has not been equivocally defined. Cha et al, ${ }^{(8)}$ evaluated the surface roughness of brackets using a scanning electron microscope and found that the same type of MCA ceramic bracket used in this study had the smoothest slot surface although it revealed the highest friction forces in all bracket-wire angulations included in their experiment. Choi et al, ${ }^{(23)}$ quantitatively measured the slot surface roughness of their brackets and found that the same type of MCA bracket used in our study had the smoothest slot surface but also the highest friction values. Similarly, the surface roughness of the bracket slots was measured via laser specular reflectance. Monocrystalline ceramic brackets were significantly smoother than Polycrystalline ceramic brackets, but there were no differences in their frictional characteristics ${ }^{(17)}$, an observation attributed to the fact that MCA ceramic brackets have sharp, hard corners formed by the intersection of the slot and side walls of the bracket, compromising the binding between the archwire and the bracket corners, which may negate any advantage associated with their smoother surfaces. We concur with this explanation ${ }^{(17)}$.

The composition of the slot is perhaps the most important factor, since the coefficient of friction, which is specific for each pair of materials, depends on it ${ }^{(24)}$. The present study shows results similar to those of other investigators, who point to SS brackets as the ones producing the lowest frictional forces $(13,14,21,25)$. This is attributable to the physical properties of the metal, which provide a low coefficient of friction and allow a good surface finish. For this reason, one of the methods used by manufacturers to improve friction levels in ceramic brackets is to incorporate metal slots. Many studies show that ceramic brackets with SS slots have superior frictional qualities compared with those of conventional ceramic; however, they are not as efficient as metal brackets ${ }^{(14,25,26)}$. Nonetheless, in another study, friction values for brackets with metal slots were similar to those of conventional ceramic brackets $^{(24)}$.Accordingly, it was recommended to use PC brackets only if they had a metal slot in order to decrease the frictional resistance, slot deformation, and to avoid damage from the sliding movements of the arch wire ${ }^{(23,27)}$. 


\section{CONCLUSION}

Based on the results of this study the following conclusions could be drawn:

- Plastic bracket with metal slot has the least friction when compared with monocrystalline and polycrystalline ceramic brackets.

- If lower static and kinetic frictions were required during sliding of ceramic brackets along the arch wire, it is preferred to use plastic bracket with metal slot.

\section{REFERENCES}

1. C. A. Reicheneder, U. Baumert, T. Gedrange , P. Proff, A. Faltermeier and D. Muessig Frictional properties of aesthetic brackets. The European Journal of Orthodontics, 2007, 29.4: 359-365.

2. Holt M H , Nanda R S , Duncanson M G. Fracture resistance of ceramic brackets during arch wire torsion. American Journal of Orthodontics and Dentofacial Orthopedics 1991;99: 287 - 293

3. Kusy R P and Whitley J Q. Friction between different wire-bracket configurations and materials. Seminars in Orthodontics 1997;3: $166-177$

4. Arici N. Akdeniz BS. Arici S. Comparison of the frictional characteristics of aesthetic orthodontic brackets measured using a modified in vitro technique. Korean J Orthod 2015;45(1):29-37

5. Pillai AR, Gangadharan A, Kumar S, Shah A. Comparison of the frictional resistance between arch wire and different bracket system: An in vitro study.J Pharm Bioallied Sci. 2014;6(Suppl 1):S150-5.

6. Oliver CL, Daskalogiannakis J, Tompson BD. Arch wire depth is a significant parameter in the frictional resistance of active and interactive, but not passive, self-ligating brackets. Angle Orthod. 2011;81(6):1036-44

7. Bazakidou E, Nanda RS, Duncanson MG Jr, Sinha P. Evaluation of frictional resistance in esthetic brackets. Am J Orthod Dentofacial Orthop 1997;112: 138-44.

8. Cha JY, Kim KS, Hwang CJ. Friction of conventional and silica-insert ceramic brackets in various bracket-wire combinations. Angle Orthod 2007; 77:100-7

9. Arash V, Rabiee M, Rakhshan V, Khorasani S, Sobouti F. In vitro evaluation of frictional forces of two ceramic orthodontic brackets versus a stainless-steel bracket in combination with two types of arch wires. J Orthod Sci. 2015;4(2):42-6.10

10. Kannan MS, Murali RV, Kishore Kumar S, Gnana Shanmugam K, Jayanth V.Comparison of frictional resistance of esthetic and semi-esthetic self-ligating brackets ..J Pharm Bioallied Sci. 2015 ;7(Suppl 1):S116-20.

11. Huffman DJ, Way DC. A clinical evaluation of tooth movement along arch wires of two different sizes. Am J Orthod. 1983;83(6):453-9.

12. Kaur G, Goyal S, Rajpal S, Gera A. Comparative evaluation of frictional forces of conventional and self-ligating bracket systems: An in vitro study. J Indian Orthod Soc 2013;47(4):211-6.

13. Yousif, Atia A.; Abdel Karim, Usama M. Assessment of friction resistance of four orthodontic arch wires using three ligation methods in dry and wet conditions. Egyptian Orthodontic Journal, 2020, 50.December 2016: 85-102.

14. Vittorio Cacciafesta, Maria Francesca Sfondrini, Andrea Ricciardi, Andrea Scribante, Catherine Klersy, Ferdinando Auricchio. Evaluation of friction of stainless steel and esthetic self-ligating brackets in various bracket-archwire combinations. American Journal of Orthodontics and Dentofacial Orthopedics, 2003, 124.4: 395-402.

15. Roberta Ferreira Pimentel 1, Roberto Sotto Maior Fortes de Oliveira, Maria das Graças Afonso Miranda Chaves, Carlos Nelson Elias, Marco Abdo Gravina. Evaluation of the friction force generated by monocristalyne and policristalyne ceramic brackets in sliding mechanics. Dental press journal of orthodontics, 2013, 18.1: 121-127.

16. Benjamin T Pliska, Rick W Fuchs, John P Beyer, Brent E Larson. Effect of applied moment on resistance to sliding among esthetic self-ligating brackets. The Angle Orthodontist, 2014, 84.1: 134-139.

17. Mai Alsubaie, Nabeel F Talic, Ahmad Alobeid. Study of force loss due to friction comparing two ceramic brackets during sliding tooth movement. Journal of Orofacial Orthopedics/Fortschritte der Kieferorthopädie, 2016, 77.5: 334-340

18. E Bazakidou 1, R S Nanda, M G Duncanson Jr, P Sinha. Evaluation of frictional resistance in esthetic brackets. American Journal of Orthodontics and Dentofacial Orthopedics, 1997, 112.2: 138-144.

19. SAUNDERS, C. R.; KUSY, Robert P. Surface topography and frictional characteristics of ceramic brackets. American Journal of Orthodontics and Dentofacial Orthopedics, 1994, 106.1: 76-87. 
20. DOSHI, Umal H.; BHAD-PATIL, Wasundhara A. Static frictional force and surface roughness of various bracket and wire combinations. American Journal of Orthodontics and Dentofacial Orthopedics, 2011, 139.1: 74-79.

21. Clarice Nishio, Andréa Fonseca Jardim da Motta, Carlos Nelson Elias, José Nelson Mucha. In vitro evaluation of frictional forces between archwires and ceramic brackets. American journal of orthodontics and dentofacial orthopedics, 2004, 125.1: 56-64.

22. WILLIAMS, Claire L.; KHALAF, Khaled. Frictional resistance of three types of ceramic brackets. Journal of oral \& maxillofacial research, 2013, 4.4.

23. CHOI, Sung-Hwan; KANG, Da-Young; HWANG, Chung-Ju. Surface roughness of three types of modern plastic bracket slot floors and frictional resistance. The Angle Orthodontist, 2014, 84.1: 177-183.
24. Ariana Pulido Guerrero, Odilon Guariza Filho, Orlando Tanaka, Elisa Souza Camargo, Sérgio Vieira. Evaluation of frictional forces between ceramic brackets and archwires of different alloys compared with metal brackets. Brazilian oral research, 2010, 24.1: 40-45.

25. Downing A, McCabe J, Gordon P. A study of frictional forces between orthodontic brackets and archwires. $\mathrm{Br} \mathrm{J}$ Orthod. 1994 Apr;21(4):349-57.

26. Loftus BP, Artur J, Nicholls JI, Alonzo TA, Stoner JA. Evaluation of friction during sliding tooth movement in various bracket-arch wire combinations. Am J Orthod DentofacialOrthop. 1999 Sep;116(3):336-45.

27. Reza Sadat-Khonsari, Azita Moshtaghy, Volker Schlegel, Bärbel Kahl-Nieke, Mathias Möller, Oskar Bauss. Torque deformation characteristics of plastic brackets: a comparative study. Journal of Orofacial Orthopedics/Fortschritte der Kieferorthopädie, 2004, 65.1: 26-33. 